\title{
Max Plus Algebra and Petri Net Application on Scheduling of Ship Engine Component's Spare Part Ordering
}

\author{
Farah Azizah and Subiono
}

\begin{abstract}
Shipping company is a company that runs its business by operating the ships or other businesses that are closely related to the ship. A ship has a main engine and some auxiliary engines to support the ship performance. It needs to do maintenance of engines so that the ship can operate properly. This engine maintenance is replacement of the old engine components with the new ones if the running hours of the components are over. Therefore, in the ship, the spare parts must always be available at least one for each engine component. During this time, the company has experienced a difficulty in determining the time of spare part ordering. When the running hours of engine components are over, the spare parts were not yet available. Then, Petri Net and Max Plus Algebra model will be built to schedule the ordering of ship engine component's spare part based on the ordering flow and the running hours of engine components. The Petri Net based on the Max Plus Algebra obtains maximum time to order the spare part so that it produces the ship engine component's spare part ordering schedule in running hour form and date. Therefore, spare part of each ship engine component is always available so that the installation can be timely and never be late.

Index Terms-Max-plus algebra, Petri nets, Scheduling of spare part ordering.
\end{abstract}

\section{INTRODUCTION}

$\mathbf{M}$ AX plus algebra is the useful approach to represent the discrete event systems. This approach makes us possible to determine and analyze various kinds of system properties. Therefore, the model of these ones will be linear over max plus algebra. But in conventional algebra, it is not linear. We can analyze the systems in max plus algebra easier and simpler than the conventional systems because of this linearity [1]. One of applications of max plus algebra is a scheduling of crystal sugar production system [2].

A Petri net is a mathematical modeling tool which can be applied to represent the state evolution of the discrete event systems. Petri net is called autonomous if every transition in this Petri net has at least an input state. This means that there is no transition which is enabled without any condition. In other words, autonomous Petri net does not have a transition which is always enabled [3]. Timed Petri net is an extension of Petri net. In this paper, we use the autonomous timed Petri net and max plus algebra model for scheduling of ship engine components' spare part ordering. Furthermore, we will

Manuscript received May 17, 2017; accepted September 19, 2017.

The authors are with the Department of Mathematics, Institut Teknologi Sepuluh Nopember, Kampus ITS Sukolilo-Surabaya 60111, Indonesia. Emails: farahazizah19@yahoo.co.id, subiono2008@matematika.its.ac.id build a model of Max Plus Algebra using Supply Chain model to obtain the date of spare part ordering. For more detailed discussion of supply chain using max plus algebra, the interested reader is referred to [4].

Shipping company is a company that runs its business by operating the ships or other businesses that are closely related to the ship. The ship becomes a very important part in this company. Therefore, the company must maintain the performance of the ship so that operations run optimally. The most important thing in keeping the performance of the ship is to make sure all of the ship's engine run properly so as not to cause delays in shipping time. Inside the ship there are two large groups of machines, the main engine and auxiliary engine. In order for ship engines to function normally, the system required periodic maintenance. Thus, the ship's engine does not get a breakdown. Periodic maintenance is generally in the form of checking up the replacement of components in the engine according to running hours of the components. Therefore, in the ship must always be available at least one each of component parts for the engine, so that when it is needed in the periodic maintenance, the spare parts can be used directly without disturbing the shipping schedule.

The spare part of each ship engine component is ordered from various suppliers. When the running hours of ship engine components will end, the ship crew will start to require the spare parts. Furthermore, the request will be processed by purchasing division until the spare parts are ready to be sent to the warehouse. Purchasing division will inform the spare parts requirement to suppliers who already have a working relationship with the company. The suppliers will offer the spare parts requested by different price, time availability, and quality. From some offers, purchasing division makes a summary of the offers which will be submitted to the ship manager to determine which supplier will be chosen. Following an agreement, the chosen supplier will provide the spare parts and send to the company's warehouse in a given time period. Thus, the spare part is available and ready to be sent to the ship.

During this time the company experienced difficulties in determining the time of spare parts ordering. When running hours of engine components are over, the spare parts are not available. As a result, the spare parts must be ordered from within the country or abroad and delivered by plane. Thus, the purchase cost is increasing. Meanwhile, the company expects the spare parts are always available before running hours of ship engine components end so that the ship can operate 
optimally. In addition, the purchase cost of spare parts become cheaper if ordering in the right time and not in a hurry because it can be delivered by land or sea transportation. Therefore, Petri Net and Max Plus Algebra model will be built to schedule the ordering of ship engine component's spare part based on the ordering flow and the running hour of engine components. It is expected that the spare part of each ship engine component is always available so that the installation can be timely.

\section{PRELIMINARIES}

\section{A. Max Plus Algebra}

Given $\mathbb{R}_{\varepsilon} \stackrel{\text { def }}{=} \mathbb{R} \cup\{\varepsilon\}$ where $\mathbb{R}$ is a set of real numbers and $\varepsilon \stackrel{\text { def }}{=}-\infty$. In $\mathbb{R}_{\varepsilon}$, two operations are defined by:

$$
x \oplus y \stackrel{\text { def }}{=} \max \{x, y\} \quad \text { and } \quad x \otimes y \stackrel{\text { def }}{=} x+y, \quad \forall x, y \in \mathbb{R}_{\varepsilon} .
$$

Furthermore, $\left(\mathbb{R}_{\mathcal{\varepsilon}}, \oplus, \otimes\right)$ is a semiring with neutral element $\varepsilon$ and unit element $e \stackrel{\text { def }}{=} 0$. For $x \in \mathbb{R}_{\varepsilon}$ and $n \in \mathbb{N}$ with $n \neq 0$, where $\mathbb{N}$ is the set of all positive integers, we define

$$
x \otimes n \stackrel{\text { def }}{=} \underbrace{x \otimes x \otimes \cdots \otimes x}_{n},
$$

whereas for $n=0$, it is defined by $x \otimes n \stackrel{\text { def }}{=} e(=0)$. Therefore, for each $n \in \mathbb{N}, x^{\otimes n}$ is defined by

$$
x \stackrel{\otimes}{ } \stackrel{\text { def }}{=} \underbrace{x \otimes x \otimes \cdots \otimes x}_{n}=n \otimes x .
$$

Furthermore,

$$
x^{\otimes \alpha}=\alpha \otimes x, \quad \text { for } \alpha \in \mathbb{R} .
$$

Adddition and multiplication of two matrices with appropriate size over max-plus algebra are defined by

$$
\begin{aligned}
& {[A \oplus B]_{i j}=[A]_{i j} \oplus[B]_{i j}} \\
& {[A \otimes B]_{i j}=\bigoplus_{k}\left([A]_{i k} \otimes[B]_{k j}\right)}
\end{aligned}
$$

where $A$ and $B$ are matrices of appropriate dimension. As an example, the multiplication of two matrices of size $2 \times 2$ is given by

$$
\left[\begin{array}{ll}
a & b \\
c & d
\end{array}\right] \otimes\left[\begin{array}{ll}
e & f \\
g & h
\end{array}\right]=\left[\begin{array}{ll}
a \otimes e \oplus b \otimes g & a \otimes f \oplus b \otimes h \\
c \otimes e \oplus d \otimes g & c \otimes f \oplus d \otimes h
\end{array}\right]
$$

and the identity matrix of size $2 \times 2$ over max plus algebra is

$$
\left[\begin{array}{ll}
0 & \varepsilon \\
\varepsilon & 0
\end{array}\right] \text {. }
$$

\section{B. Petri Nets}

Definition 1 ([1]): Petri net is 4-tuple $(P, T, A, w)$ with

- $P$ : a finite set of places, $P=\left\{p_{1}, p_{2}, \ldots, p_{n}\right\}$;

- $T$ : a finite set of transitions, $T=\left\{t_{1}, t_{2}, \ldots, t_{n}\right\}$;

- $A$ : a set of arcs, $A \subseteq(P \times T) \cup(T \times P)$;

- $w$ : a weight function, $w \rightarrow\{1,2,3, \ldots\}$.

Definition 2 ([1]): Marking $x$ in a Petri net is a function $x: P \rightarrow\{0,1,2, \ldots\}$.

Definition 3: Transition $t_{j} \in T$ in a Petri net is enabled if $x\left(p_{i}\right) \geq w\left(p_{i}, t_{j}\right)$, for all $p_{i} \in I\left(t_{j}\right)$.

\section{Prioritized Petri Nets}

Each transition in a Petri net has a priority value and denoted by $\pi$. Prioritized Petri Net is a Petri Net which prioritizes one or several transition(s) so that in certain condition, the prioritized transition is selected from a set of enabled transitions. This situation is shown in Fig. 1 and 2 .

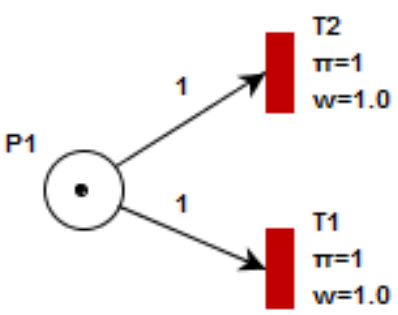

Fig. 1. Petri Net which has two transitions with the same priority.

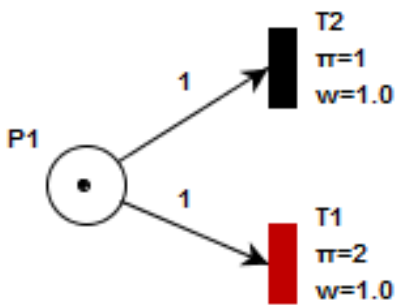

Fig. 2. Petri Net which has two transitions with different priority.

Figure 1 shows that the transition $T_{1}$ and $T_{2}$ is equally enabled and has a priority value $\pi=1$. Meanwhile, in Fig. 2, the transition $T_{2}$ is enabled, but the transition $T_{1}$ is not enabled. This condition is caused by the priority value of transition $T_{2}$ is higher than the priority value of transition $T_{1}$. Thus, only transition $T_{2}$ can be fired.

\section{System of Ship Engine Component's Spare Part Ordering}

The flow of ship engine component's spare part ordering is described in Fig. 3. Furthermore, the process of ship engine

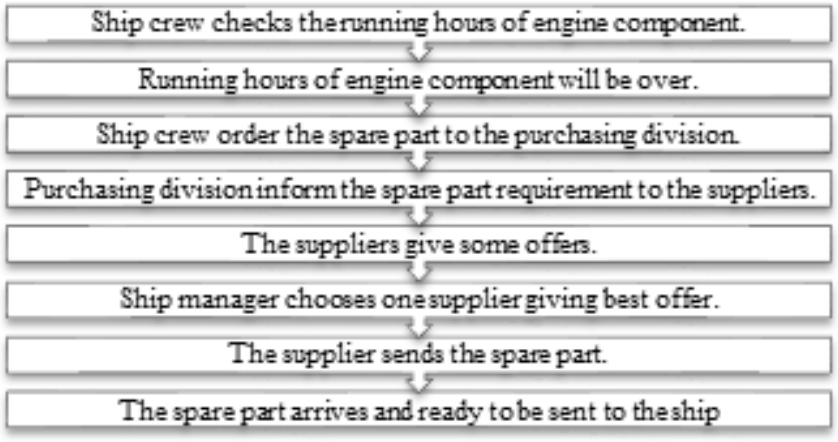

Fig. 3. Flow Chart of Ship Engine Component's Spare Part Ordering.

component's spare part ordering will be represented by a Petri Net and use it to construct a Max Plus Algebra model for obtaining the maximal time of spare part ordering. 


\section{RESUlTS AND Discussions}

\section{A. Model of Ship Engine Component' Spare Part Ordering in Max Plus Algebra}

Based on Fig. 3, we obtain Petri Net on Fig. 4 that represents the process of ship engine component's spare part ordering, where the interpretation of transitions is as follows:

- $T_{1}$ : spare part ordering by the ship's crew to purchasing division $(\pi=1)$.

- $T_{2}$ : dissemination of ordering information by purchasing division to suppliers $(\pi=2)$.

- $T_{3}$ : offering from suppliers $(\pi=2)$.

- $T_{4}$ : manager checks the offering summary from suppliers $(\pi=2)$.

- $T_{5}$ : the offering of type A $(\pi=8)$.

- $T_{6}$ : the offering of type $\mathrm{A}$ is not available or ignored $(\pi=8)$.

- $T_{7}$ : the offering of type $B(\pi=7)$.

- $T_{8}$ : the offering of type $\mathrm{B}$ is not available or ignored $(\pi=7)$.

- $T_{9}$ : the offering of type $\mathrm{C}(\pi=6)$.

- $T_{10}$ : the offering of type $\mathrm{C}$ is not available or ignored $(\pi=6)$.

- $T_{11}$ : the offering of type $\mathrm{D}(\pi=5)$.

- $T_{12}$ : the offering of type $\mathrm{D}$ is not available or ignored $(\pi=4)$.

- $T_{13}$ : the offering of type $\mathrm{A}$ is picked $(\pi=3)$.

- $T_{14}$ : the offer other than type A is picked $(\pi=2)$.

- $T_{15}$ : the offer rejected $(\pi=2)$.

- $T_{16}$ : manager instruct the re-dissemination of information offering by purchasing division to other suppliers $(\pi=2)$.

- $T_{17}$ : spare parts start to order $(\pi=2)$.

- $T_{18}$ : spare parts arrived at the warehouse $(\pi=2)$.

The meaning of each place is given by

- $P_{1}$ : purchasing division receives spare parts ordering

- $P_{2}$ : suppliers receive ordering information from purchasing division

- $P_{3}$ : the offering summary is submitted to the manager by purchasing division

- $P_{4}$ : the offering of type $\mathrm{A}$

- $P_{5}$ : the offering of type $\mathrm{B}$

- $P_{6}$ : the offering of type $\mathrm{C}$

- $P_{7}$ : the offering of type $\mathrm{D}$

- $P_{8}$ : a decision on the acceptance or rejection of the offering

- $P_{9}$ : saving temporary offering summary that is rejected

- $P_{10}$ : the offering of type $\mathrm{A}$ is prioritized

- $P_{11}:$ selected one supplier

- $P_{12}$ : selected suppliers provide spare parts ordered

Furthermore, this Petri Net is used to make the following Max Plus Algebra model which produces maximum time of ship engine component's spare part ordering.

$$
\begin{aligned}
& T_{1}(k)=v_{T_{1}, k} \otimes T_{1}(k-1) \\
& T_{2}(k)=v_{T_{2}, k} \otimes T_{1}(k) \oplus v_{T_{2}, k} \otimes T_{16}(k-1) \\
& T_{3}(k)=v_{T_{3}, k} \otimes T_{2}(k) \\
& T_{4}(k)=v_{T_{4}, k} \otimes T_{3}(k)
\end{aligned}
$$

$$
\begin{aligned}
T_{5}(k) & =v_{T_{5}, k} \otimes T_{4}(k) \\
T_{6}(k) & =v_{T_{6}, k} \otimes T_{4}(k) \\
T_{7}(k) & =v_{T_{7}, k} \otimes T_{4}(k) \\
T_{8}(k) & =v_{T_{8}, k} \otimes T_{4}(k) \\
T_{9}(k) & =v_{T_{9}, k} \otimes T_{4}(k) \\
T_{10}(k) & =v_{T_{10}, k} \otimes T_{4}(k) \\
T_{11}(k) & =v_{T_{11}, k} \otimes T_{4}(k) \\
T_{12}(k) & =v_{T_{12}, k} \otimes T_{4}(k) \\
T_{13}(k) & =v_{T_{13}, k} \otimes\left[T_{5}(k) \oplus T_{7}(k) \oplus T_{9}(k) \oplus T_{11}(k)\right] \\
T_{14}(k) & =v_{T_{14}, k} \otimes\left[T_{5}(k) \oplus T_{7}(k) \oplus T_{9}(k) \oplus T_{11}(k)\right] \\
T_{15}(k) & =v_{T_{15}, k} \otimes\left[T_{5}(k) \oplus T_{7}(k) \oplus T_{9}(k) \oplus T_{11}(k)\right] \\
T_{16}(k) & =v_{T_{16}, k} \otimes T_{15}(k) \\
T_{17}(k) & =v_{T_{17}, k} \otimes\left[T_{13}(k) \oplus T_{14}(k)\right] \\
T_{18}(k) & =v_{T_{18}, k} \otimes T_{17}(k)
\end{aligned}
$$

Based on equation (1), (16), and (18), we obtain

$$
\left[\begin{array}{c}
T_{1}(k) \\
T_{16}(k) \\
T_{18}(k)
\end{array}\right]=\left[\begin{array}{ccc}
v_{T_{1}, k} & \varepsilon & \varepsilon \\
a & b & \varepsilon \\
c_{n} & d_{n} & \varepsilon
\end{array}\right] \otimes\left[\begin{array}{c}
T_{1}(k-1) \\
T_{16}(k-1) \\
T_{18}(k-1)
\end{array}\right]
$$

where

- $v_{T_{1}, k}$ : duration of spare part offering by crew that is accepted by purchasing division

- $v_{T_{2}, k}$ : duration of dissemination offering process from purchasing division to suppliers

- $v_{T_{3}, k}$ : duration of offering from the first supplier since receiving the request information

- $v_{T_{4}, k}$ : duration of offering from the second supplier since receiving the request information

- $v_{T_{5}, k}$ : duration of offering from the third supplier since receiving the request information

- $v_{T_{6}, k}$ : duration of offering from the fourth supplier since receiving the request information

- $v_{T_{7}, k}$ : duration submission of offering summary to the manager

- $v_{T_{8}, k}$ : duration of the manager determines all offerings being rejected

- $v_{T_{9}, k}$ : duration of offering from other suppliers since receiving the information

- $v_{T_{10}, k}$ : duration of submitting a new offering summary to manager

- $v_{T_{11}, k}$ : duration of the manager determines one of the offerings received

- $v_{T_{12}, k}$ : duration of spare parts began to be ordered since supplier determination

- $v_{T_{12}, k}$ : duration of spare parts began to be ordered since the determination of selected suppliers

- $v_{T_{13}, k}$ : duration of spare parts arrived in the warehouse since it was ordered.

and

$$
\begin{gathered}
a=v_{T_{1}, k} \otimes v_{T_{2}, k} \otimes v_{T_{3}, k} \otimes v_{T_{4}, k} \otimes v_{T_{5}, k} \otimes v_{T_{15}, k} \otimes v_{T_{16}, k} \oplus \\
v_{T_{1}, k} \otimes v_{T_{2}, k} \otimes v_{T_{3}, k} \otimes v_{T_{4}, k} \otimes v_{T_{7}, k} \otimes v_{T_{15}, k} \otimes v_{T_{16}, k} \oplus \\
v_{T_{1}, k} \otimes v_{T_{2}, k} \otimes v_{T_{3}, k} \otimes v_{T_{4}, k} \otimes v_{T_{9}, k} \otimes v_{T_{15}, k} \otimes v_{T_{16}, k} \oplus \\
v_{T_{1}, k} \otimes v_{T_{2}, k} \otimes v_{T_{3}, k} \otimes v_{T_{4}, k} \otimes v_{T_{11}, k} \otimes v_{T_{15}, k} \otimes v_{T_{16}, k}
\end{gathered}
$$




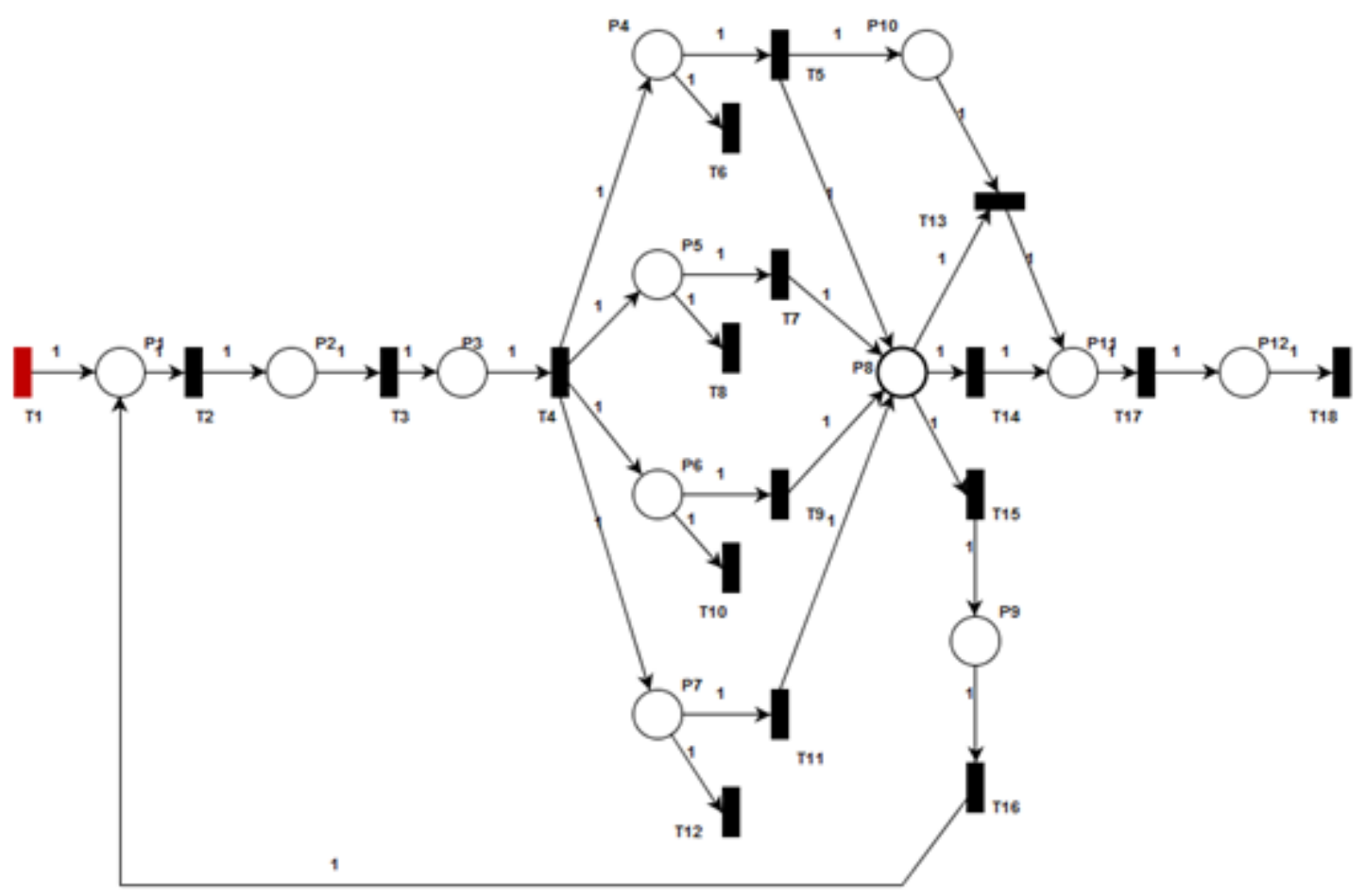

Fig. 4. Prioritized Petri Net of Engine Component's Spare Part Ordering.

$$
\begin{gathered}
b=v_{T_{2}, k} \otimes v_{T_{3}, k} \otimes v_{T_{4}, k} \otimes v_{T_{5}, k} \otimes v_{T_{15}, k} \otimes v_{T_{16}, k} \oplus \\
v_{T_{2}, k} \otimes v_{T_{3}, k} \otimes v_{T_{4}, k} \otimes v_{T_{7}, k} \otimes v_{T_{15}, k} \otimes v_{T_{16}, k} \oplus \\
v_{T_{2}, k} \otimes v_{T_{3}, k} \otimes v_{T_{4}, k} \otimes v_{T_{9}, k} \otimes v_{T_{15}, k} \otimes v_{T_{16}, k} \oplus \\
v_{T_{2}, k} \otimes v_{T_{3}, k} \otimes v_{T_{4}, k} \otimes v_{T_{11}, k} \otimes v_{T_{15}, k} \otimes v_{T_{16}, k} \\
c_{n}=v_{T_{1}, k} \otimes\left(v_{T_{2}, k} \otimes v_{T_{3}, k} \otimes v_{T_{4}, k} \otimes v_{T_{5}, k}\right)^{\otimes n} \otimes \\
v_{T_{14}, k} \otimes v_{T_{15}, k} \otimes v_{T_{16}, k} \otimes v_{T_{17}, k} \otimes v_{T_{18}, k} \oplus \\
v_{T_{1}, k} \otimes\left(v_{T_{2}, k} \otimes v_{T_{3}, k} \otimes v_{T_{4}, k} \otimes v_{T_{7}, k}\right)^{\otimes n} \otimes \\
v_{T_{14}, k} \otimes v_{T_{15}, k} \otimes v_{T_{16}, k} \otimes v_{T_{17}, k} \otimes v_{T_{18}, k} \oplus \\
v_{T_{1}, k} \otimes\left(v_{T_{2}, k} \otimes v_{T_{3}, k} \otimes v_{T_{4}, k} \otimes v_{T_{9}, k}\right)^{\otimes n} \otimes \\
v_{T_{14}, k} \otimes v_{T_{15}, k} \otimes v_{T_{16}, k} \otimes v_{T_{17}, k} \otimes v_{T_{18}, k} \oplus \\
v_{T_{1}, k} \otimes\left(v_{T_{2}, k} \otimes v_{T_{3}, k} \otimes v_{T_{4}, k} \otimes v_{T_{11}, k}\right)^{\otimes n} \otimes \\
v_{T_{14}, k} \otimes v_{T_{15}, k} \otimes v_{T_{16}, k} \otimes v_{T_{17}, k} \otimes v_{T_{18}, k} \\
d_{n}=\left(v_{T_{2}, k} \otimes v_{T_{3}, k} \otimes v_{T_{4}, k} \otimes v_{T_{5}, k}\right)^{\otimes n} \otimes \\
v_{T_{14}, k} \otimes v_{T_{15}, k} \otimes v_{T_{16}, k} \otimes v_{T_{17}, k} \otimes v_{T_{18}, k} \oplus \\
\left(v_{T_{2}, k} \otimes v_{T_{3}, k} \otimes v_{T_{4}, k} \otimes v_{T_{7}, k}\right)^{\otimes n} \otimes \\
v_{T_{14}, k} \otimes v_{T_{15}, k} \otimes v_{T_{16}, k} \otimes v_{T_{17}, k} \otimes v_{T_{18}, k} \oplus \\
\left(v_{T_{2}, k} \otimes v_{T_{3}, k} \otimes v_{T_{4}, k} \otimes v_{T_{9}, k}\right)^{\otimes n} \otimes \\
v_{T_{14}, k} \otimes v_{T_{15}, k} \otimes v_{T_{16}, k} \otimes v_{T_{17}, k} \otimes v_{T_{18}, k} \oplus \\
\left(v_{T_{2}, k} \otimes v_{T_{3}, k} \otimes v_{T_{4}, k} \otimes v_{T_{11}, k}\right)^{\otimes n} \otimes \\
v_{T_{14}, k} \otimes v_{T_{15}, k} \otimes v_{T_{16}, k} \otimes v_{T_{17}, k} \otimes v_{T_{18}, k}
\end{gathered}
$$

and $n$ is the number of spare part requirement information spreading to the suppliers. Therefore, for $n=2$, we obtained

$$
c_{2}=39 \otimes v_{T_{18}, k}
$$

$$
d_{2}=38 \otimes v_{T_{18}, k}
$$

The maximal time of spare part ordering from equation (19) is in day unit so it has to be converted to hour unit by multiplying it with 10 hours which is the average of ship's running hours every day, then we get the ordering duration. To compute the time when the spare part starts to be ordered, we have to subtract the interval for overhaul by the ordering duration and the result is showed in Table I.

Furthermore, we will construct a model of Max Plus Algebra using Supply Chain model to obtain the date of spare part ordering. Figure 5 represents the process of spare part ordering using a Supply Chain model.

Based on Fig. 5, we get the following Max Plus Algebra model:

$$
\begin{aligned}
t_{2}(k) & =w_{a} \otimes t_{3}(k-n) \oplus t_{1}(k) \\
t_{3}(k) & =w_{b} \otimes t_{2}(k) \\
y(k) & =t_{3}(k)
\end{aligned}
$$

where $n=1$ is the number of spare parts.

The model can be changed into matrix equations as follow.

$$
\begin{aligned}
& X(k)=A_{0} \otimes X(k) \oplus A_{1} \otimes X(k-n) \oplus B_{0} \otimes U(k) \\
& Y(k)=C \otimes X(k)
\end{aligned}
$$

where

$$
\begin{aligned}
& U(k)=\left[t_{1}(k)\right] \\
& X(k)=\left[\begin{array}{l}
t_{2}(k) \\
t_{3}(k)
\end{array}\right]
\end{aligned}
$$




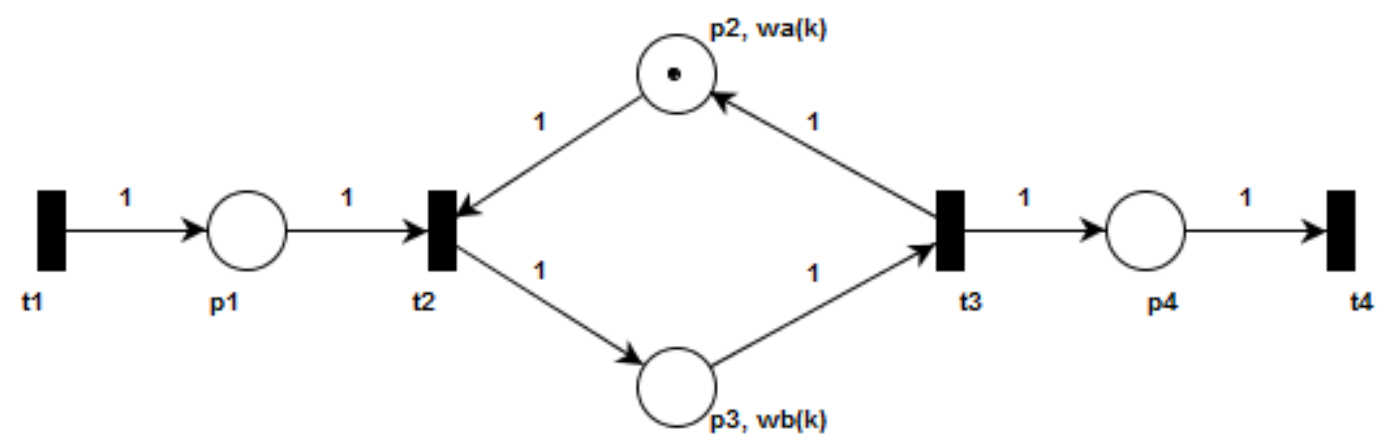

Fig. 5. Petri Net of Spare Part Ordering Using Supply Chain Model.

$$
\begin{aligned}
Y(k) & =\left[t_{4}(k)\right] \\
A_{0} & =\left[\begin{array}{cc}
\varepsilon & \varepsilon \\
w_{b} & \varepsilon
\end{array}\right] \\
A_{1} & =\left[\begin{array}{cc}
\varepsilon & w_{a} \\
\varepsilon & \varepsilon
\end{array}\right] \\
B_{0} & =\left[\begin{array}{l}
0 \\
\varepsilon
\end{array}\right] \\
C & =\left[\begin{array}{ll}
\varepsilon & 0
\end{array}\right]
\end{aligned}
$$

Since $A=A_{0}{ }^{*} \otimes A_{1}$ and $B=A_{0}{ }^{*} \otimes B_{0}[3]$, then

$$
\begin{aligned}
A & =A_{0}{ }^{*} \otimes A_{1} \\
& =\left[\begin{array}{cc}
0 & \varepsilon \\
w_{b} & 0
\end{array}\right] \otimes\left[\begin{array}{cc}
\varepsilon & w_{a} \\
\varepsilon & \varepsilon
\end{array}\right] \\
& =\left[\begin{array}{cc}
0 \otimes \varepsilon \oplus \varepsilon \otimes \varepsilon & 0 \otimes w_{a} \oplus \varepsilon \otimes \varepsilon \\
w_{b} \otimes \varepsilon \oplus 0 \otimes \varepsilon & w_{b} \otimes w_{a} \oplus 0 \otimes \varepsilon
\end{array}\right] \\
& =\left[\begin{array}{cc}
\varepsilon & w_{a} \\
\varepsilon & w_{b} \otimes w_{a}
\end{array}\right] \\
& =\left[\begin{array}{cc}
\varepsilon & w_{a} \\
\varepsilon & w_{a} w_{b}
\end{array}\right]
\end{aligned}
$$

and

$$
\begin{aligned}
B & =A_{0}{ }^{*} \otimes B_{0} \\
& =\left[\begin{array}{cc}
0 & \varepsilon \\
w_{b} & 0
\end{array}\right] \otimes\left[\begin{array}{l}
0 \\
\varepsilon
\end{array}\right] \\
& =\left[\begin{array}{c}
0 \otimes 0 \oplus \varepsilon \otimes \varepsilon \\
w_{b} \otimes 0 \oplus 0 \otimes \varepsilon
\end{array}\right] \\
& =\left[\begin{array}{c}
0 \oplus \varepsilon \\
w_{b} \oplus \varepsilon
\end{array}\right] \\
& =\left[\begin{array}{c}
0 \\
w_{b}
\end{array}\right]
\end{aligned}
$$

so that

$$
\begin{aligned}
C B & =\left[\begin{array}{ll}
\varepsilon & 0
\end{array}\right] \otimes\left[\begin{array}{c}
0 \\
w_{b}
\end{array}\right] \\
& =\left[\varepsilon \otimes 0 \oplus 0 \otimes w_{b}\right]
\end{aligned}
$$

$$
\begin{aligned}
& =\left[\varepsilon \oplus w_{b}\right] \\
& =\left[w_{b}\right]
\end{aligned}
$$

and

$$
\begin{aligned}
C A B & =\left[\begin{array}{ll}
\varepsilon & 0
\end{array}\right] \otimes\left[\begin{array}{cc}
\varepsilon & w_{a} \\
\varepsilon & w_{a} w_{b}
\end{array}\right] \otimes\left[\begin{array}{c}
0 \\
w_{b}
\end{array}\right] \\
& =\left[\begin{array}{cc}
\varepsilon \otimes \varepsilon \oplus 0 \otimes \varepsilon & \varepsilon \otimes w_{a} \oplus 0 \otimes w_{a} w_{b}
\end{array}\right] \otimes\left[\begin{array}{c}
0 \\
w_{b}
\end{array}\right] \\
& =\left[\begin{array}{cc}
\varepsilon \oplus \varepsilon & \varepsilon \oplus w_{a} w_{b}
\end{array}\right] \otimes\left[\begin{array}{c}
0 \\
w_{b}
\end{array}\right] \\
& =\left[\begin{array}{cc}
\varepsilon & w_{a} w_{b}
\end{array}\right] \otimes\left[\begin{array}{c}
0 \\
w_{b}
\end{array}\right] \\
& =\left[\varepsilon \otimes 0 \oplus w_{a} w_{b} \otimes w_{b}\right] \\
& =\left[\begin{array}{ll}
\varepsilon \oplus w_{a} w_{b}{ }^{\otimes 2}
\end{array}\right] \\
& =\left[\begin{array}{ll}
w_{a} w_{b}{ }^{\otimes 2}
\end{array}\right]
\end{aligned}
$$

According to [1]

$$
y(k)=\bigoplus_{i=0}^{\alpha} C \otimes A^{\otimes i} \otimes B \otimes u(k-i \cdot n)
$$

so that for $n=1$ and $\alpha=[k / n]=[k]=k$, we obtain

$$
\begin{aligned}
y(1)= & C \otimes A^{\otimes 0} \otimes B \otimes u(1-0 \cdot 1) \oplus C \otimes A^{\otimes 1} \otimes B \otimes u(1-1 \cdot 1) \\
= & C \otimes B \otimes u(1) \oplus C \otimes A^{\otimes 1} \otimes B \otimes u(1-1 \cdot 1) \\
= & C \otimes B \otimes u(1) \oplus C \otimes A \otimes B \otimes u(0) \\
y(2)= & \left.C \otimes A^{\otimes 0} \otimes B \otimes u(2-0 \cdot 1) \oplus C \otimes A^{\otimes 1} \otimes B \otimes u(2-1 \cdot 1)\right) \oplus \\
& C \otimes A^{\otimes 2} \otimes B \otimes u(2-2 \cdot 1) \\
= & C \otimes B \otimes u(2) \oplus C \otimes A^{\otimes 1} \otimes B \otimes u(1) \oplus C \otimes A^{\otimes 2} \otimes B \otimes u(0) \\
= & C \otimes B \otimes u(2) \oplus C \otimes A \otimes B \otimes u(1)
\end{aligned}
$$

Therefore, for number of ordering $l=1,2$, we obtain

$$
Y=H \otimes U
$$


where

$$
\begin{aligned}
& Y=\left[\begin{array}{l}
y(1) \\
y(2)
\end{array}\right], \\
& H=\left[\begin{array}{cc}
C B & \varepsilon \\
C A B & C B
\end{array}\right], \\
& U=\left[\begin{array}{l}
u(1) \\
u(2)
\end{array}\right] .
\end{aligned}
$$

Notice that $Y$ is time when the running hours of engine component over and $U$ is the time when the spare part ordering starts.

Therefore, the solution is [1]

$$
U=-H^{T} \oplus^{\prime} Y
$$

where

$$
\begin{aligned}
& u(1)=\min \left\{y_{1}-h_{1,1}, y_{2}-h_{2,1}\right\}, \\
& u(2)=\min \left\{y_{1}-h_{1,2}, y_{2}-h_{2,2}\right\} .
\end{aligned}
$$

\begin{tabular}{|c|c|c|c|}
\hline Spare part's name & $\begin{array}{c}\text { Ordering } \\
\text { duration } \\
\text { (hours) }\end{array}$ & $\begin{array}{l}\text { Overhaul } \\
\text { for } \\
\text { interval } \\
\text { (hours) }\end{array}$ & $\begin{array}{c}\text { Running } \\
\text { hours } \\
\text { when } \\
\text { the } \\
\text { ordering } \\
\text { is started } \\
\text { (hours) }\end{array}$ \\
\hline $\begin{array}{l}\text { CRANKPIN BEAR- } \\
\text { ING SHELL }\end{array}$ & 520 & 16000 & 15480 \\
\hline $\begin{array}{l}\text { CROSSHEAD } \\
\text { BEARING SHELL }\end{array}$ & 650 & & 15350 \\
\hline $\begin{array}{l}\text { O-RING } \\
\text { N17M6220 }\end{array}$ & 940 & 8000 & 7060 \\
\hline $\begin{array}{ll}\begin{array}{l}\text { SCRAPER } \\
\text { (LOWER) }\end{array} & \text { RING }\end{array}$ & 940 & & 7060 \\
\hline $\begin{array}{l}\begin{array}{l}\text { SCRAPER } \\
\text { (UPPER) }\end{array} \\
\text { RING }\end{array}$ & 940 & & 7060 \\
\hline $\begin{array}{l}\text { TIGHTENING } \\
\text { RING }\end{array}$ & 940 & & 7060 \\
\hline $\begin{array}{lll}\text { PISTON } & \text { RING } & - \\
3169804 & & \end{array}$ & 730 & 8000 & 7270 \\
\hline $\begin{array}{lll}\text { PISTON } & \text { RING } & - \\
3169805 & & \end{array}$ & 1400 & & 6600 \\
\hline GUIDE RING & 880 & 4000 & 3120 \\
\hline O-RING - 4511913 & 490 & & 3510 \\
\hline O-RING - 4511912 & 490 & & 3510 \\
\hline $\begin{array}{l}\text { O-RING } \\
\text { EN17M340 }\end{array}$ & 730 & & 3270 \\
\hline O-RING - 4183312 & 940 & & 3060 \\
\hline
\end{tabular}

\section{B. Schedule of Engine Component's Spare Part Ordering}

\begin{tabular}{|c|c|c|c|}
\hline Spare part's name & $\begin{array}{c}\text { Ordering } \\
\text { duration } \\
\text { (hours) }\end{array}$ & $\begin{array}{l}\text { Overhaul } \\
\text { for } \\
\text { interval } \\
\text { (hours) }\end{array}$ & $\begin{array}{c}\text { Running } \\
\text { hours } \\
\text { when } \\
\text { the } \\
\text { ordering } \\
\text { is started } \\
\text { (hours) }\end{array}$ \\
\hline $\begin{array}{l}\text { O-RING } \\
\text { EN17M365 }\end{array}$ & 730 & & 3270 \\
\hline PISTON RING & 730 & & 3270 \\
\hline $\begin{array}{lll}\text { SEAL } & \text { RING } \\
4184389 & & \end{array}$ & 730 & & 3270 \\
\hline $\begin{array}{lll}\text { SEAL } & \text { RING } & - \\
4184390 & & \end{array}$ & 730 & & 3270 \\
\hline SPACER RING & 730 & & 3270 \\
\hline O-RING - 4181145 & 730 & 8000 & 7270 \\
\hline O-RING - 4181146 & 940 & & 7060 \\
\hline O-RING - 4183002 & 940 & & 7060 \\
\hline SLIDE VALVE ASS & 730 & & 7270 \\
\hline $\begin{array}{ll}\text { SPINDLE } & \text { GUIDE } \\
\text { ASS } & \\
\end{array}$ & 730 & & 7270 \\
\hline SPACER RING & 490 & 16000 & 15510 \\
\hline SCRAPER RING & 730 & & 15270 \\
\hline O-RING - 4181145 & 940 & & 15060 \\
\hline O-RING - 4181452 & 730 & & 15270 \\
\hline O-RING - 4181455 & 730 & & 15270 \\
\hline SLIDE VALVE & 500 & & 15500 \\
\hline SPRING & 500 & & 15500 \\
\hline THRUST PIECE & 500 & & 15500 \\
\hline $\begin{array}{l}\text { CYLINDER COM- } \\
\text { PLETE }\end{array}$ & 540 & & 15460 \\
\hline
\end{tabular}

Based on the ordering duration and overhaul interval of each spare part which are gotten before, we can determine the running hours when the ordering is started that is shown in Table I.
TABLE I. Running Hours of Engine Component When the Spare Part Starts to Be Ordered.

Furthermore, the running hours when the ordering is started is converted into day unit by dividing it by 10 which is the average of ship's running hour every day. By using equation (20), we obtain the date when each spare part starts to be ordered that is presented on Table II until Table $\mathrm{V}$ below.

\begin{tabular}{|l|l|l|}
\hline Spare part's name & \multicolumn{2}{|c|}{$\begin{array}{l}\text { Date when spare part starts to be } \\
\text { ordered }\end{array}$} \\
\hline $\begin{array}{l}\text { CRANKPIN BEAR- } \\
\text { ING SHELL }\end{array}$ & $\begin{array}{l}11 \text { January } \\
2020\end{array}$ & 29 May 2024 \\
\hline $\begin{array}{l}\text { CROSSHEAD } \\
\text { BEARING SHELL }\end{array}$ & $\begin{array}{l}29 \text { December } \\
2019\end{array}$ & 16 May 2024 \\
\hline O-RING & $\begin{array}{l}02 \text { December } \\
2016\end{array}$ & $\begin{array}{l}10 \text { February } \\
2019\end{array}$ \\
\hline $\begin{array}{l}\text { SCRAPER RING } \\
\text { (LOWER) }\end{array}$ & $\begin{array}{l}02 \text { December } \\
2016\end{array}$ & $\begin{array}{l}10 \text { February } \\
2019\end{array}$ \\
\hline $\begin{array}{l}\text { SCRAPER RING } \\
\text { (UPPER) }\end{array}$ & 02 December & $\begin{array}{l}10 \quad \text { February } \\
2016\end{array}$ \\
\hline $\begin{array}{l}\text { TIGHTENING } \\
\text { RING }\end{array}$ & 02 December & $\begin{array}{l}10 \text { February } \\
2016\end{array}$ \\
\hline PISTON RING - & $\begin{array}{l}13 \text { October } \\
2017 \text { December } \\
\text { 3169804 }\end{array}$ & 2019 \\
\hline
\end{tabular}




\begin{tabular}{|c|c|c|}
\hline Spare part's name & \multicolumn{2}{|c|}{$\begin{array}{c}\text { Date when spare part starts to be } \\
\text { ordered }\end{array}$} \\
\hline $\begin{array}{lll}\text { PISTON } & \text { RING } & - \\
3169805 & & \end{array}$ & $\begin{array}{ll}07 & \text { August } \\
2017 & \end{array}$ & $\begin{array}{l}16 \text { October } \\
2019\end{array}$ \\
\hline GUIDE RING & $\begin{array}{lr}23 & \text { August } \\
2016 & \end{array}$ & $\begin{array}{l}27 \text { September } \\
2017\end{array}$ \\
\hline O-RING - 4511913 & $\begin{array}{l}01 \text { October } \\
2016\end{array}$ & $\begin{array}{l}05 \text { November } \\
2017\end{array}$ \\
\hline O-RING - 4511912 & $\begin{array}{l}01 \text { October } \\
2016\end{array}$ & $\begin{array}{l}05 \text { November } \\
2017\end{array}$ \\
\hline $\begin{array}{l}\text { O-RING } \\
\text { EN17M340 }\end{array}$ & $\begin{array}{l}\text { 07 September } \\
2016\end{array}$ & $\begin{array}{l}12 \text { October } \\
2017\end{array}$ \\
\hline O-RING - 4183312 & $\begin{array}{lr}17 & \text { August } \\
2016 & \end{array}$ & $\begin{array}{l}21 \text { September } \\
2017\end{array}$ \\
\hline $\begin{array}{l}\text { O-RING } \\
\text { EN17M365 }\end{array}$ & $\begin{array}{l}\text { 07 September } \\
2016\end{array}$ & $\begin{array}{ll}12 & \text { October } \\
2017 & \end{array}$ \\
\hline PISTON RING & $\begin{array}{l}\text { 07 September } \\
2016\end{array}$ & $\begin{array}{l}12 \text { October } \\
2017\end{array}$ \\
\hline $\begin{array}{lll}\text { SEAL } & \text { RING } \quad- \\
4184389 & & \end{array}$ & $\begin{array}{l}\text { 07 September } \\
2016\end{array}$ & $\begin{array}{l}12 \text { October } \\
2017\end{array}$ \\
\hline $\begin{array}{l}\text { SEAL RING } \quad- \\
4184390\end{array}$ & $\begin{array}{l}\text { 07 September } \\
2016\end{array}$ & $\begin{array}{l}12 \text { October } \\
2017\end{array}$ \\
\hline SPACER RING & $\begin{array}{l}\text { 07 September } \\
2016\end{array}$ & $\begin{array}{ll}12 & \text { October } \\
2017 & \\
\end{array}$ \\
\hline O-RING - 4181145 & $\begin{array}{l}16 \text { January } \\
2016\end{array}$ & $\begin{array}{ll}26 & \text { March } \\
2018 & \\
\end{array}$ \\
\hline O-RING - 4181146 & $\begin{array}{l}26 \text { December } \\
2015\end{array}$ & $\begin{array}{ll}05 & \text { March } \\
2018 & \end{array}$ \\
\hline O-RING - 4183002 & $\begin{array}{l}26 \text { December } \\
2015\end{array}$ & $\begin{array}{ll}05 & \text { March } \\
2018 & \end{array}$ \\
\hline SLIDE VALVE ASS & $\begin{array}{l}16 \text { January } \\
2016\end{array}$ & $\begin{array}{ll}26 & \text { March } \\
2018 & \end{array}$ \\
\hline $\begin{array}{l}\text { SPINDLE GUIDE } \\
\text { ASS }\end{array}$ & $\begin{array}{l}16 \text { January } \\
2016\end{array}$ & $\begin{array}{ll}26 & \text { March } \\
2018 & \\
\end{array}$ \\
\hline SPACER RING & $\begin{array}{l}13 \text { February } \\
2016\end{array}$ & 01 July 2020 \\
\hline SCRAPER RING & $\begin{array}{l}20 \text { January } \\
2016\end{array}$ & 07 June 2020 \\
\hline O-RING - 4181145 & $\begin{array}{l}30 \text { December } \\
2015\end{array}$ & 17 May 2020 \\
\hline O-RING - 4181452 & $\begin{array}{l}20 \text { January } \\
2016\end{array}$ & 07 June 2020 \\
\hline O-RING - 4181455 & $\begin{array}{lr}20 & \text { January } \\
2016 & \end{array}$ & 07 June 2020 \\
\hline SLIDE VALVE & $\begin{array}{l}12 \text { February } \\
2016\end{array}$ & 30 June 2020 \\
\hline SPRING & $\begin{array}{l}12 \text { February } \\
2016\end{array}$ & 30 June 2020 \\
\hline THRUST PIECE & $\begin{array}{l}12 \text { February } \\
2016\end{array}$ & 30 June 2020 \\
\hline $\begin{array}{l}\text { CYLINDER COM- } \\
\text { PLETE }\end{array}$ & $\begin{array}{l}08 \text { February } \\
2016\end{array}$ & 26 June 2020 \\
\hline
\end{tabular}

TABLE II. The date of spare part's ordering for cylinder 1.

\begin{tabular}{|c|c|c|}
\hline Spare part's name & Date when spe & $\begin{array}{l}\text { part starts to be } \\
\text { ered }\end{array}$ \\
\hline $\begin{array}{l}\text { CRANKPIN BEAR- } \\
\text { ING SHELL }\end{array}$ & $\begin{array}{ll}23 & \text { August } \\
2018 & \end{array}$ & $\begin{array}{ll}09 & \text { January } \\
2023 & \end{array}$ \\
\hline $\begin{array}{l}\text { CROSSHEAD } \\
\text { BEARING SHELL }\end{array}$ & $\begin{array}{lr}10 & \text { August } \\
2018 & \end{array}$ & $\begin{array}{l}27 \text { December } \\
2022\end{array}$ \\
\hline O-RING & 05 May 2016 & 14 July 2018 \\
\hline $\begin{array}{ll}\begin{array}{l}\text { SCRAPER } \\
\text { (LOWER) }\end{array} & \text { RING }\end{array}$ & 05 May 2016 & 14 July 2018 \\
\hline $\begin{array}{l}\text { SCRAPER } \\
\text { (UPPER) }\end{array}$ & 05 May 2016 & 14 July 2018 \\
\hline $\begin{array}{l}\text { TIGHTENING } \\
\text { RING }\end{array}$ & 05 May 2016 & 14 July 2018 \\
\hline $\begin{array}{lll}\text { PISTON } & \text { RING } & - \\
3169804 & & \end{array}$ & $\begin{array}{lr}04 & \text { August } \\
2018 & \end{array}$ & $\begin{array}{l}12 \text { October } \\
2020\end{array}$ \\
\hline $\begin{array}{lll}\text { PISTON } & \text { RING } & - \\
3169805 & & \end{array}$ & 29 May 2018 & $\begin{array}{ll}06 & \text { August } \\
2020 & \end{array}$ \\
\hline GUIDE RING & 06 July 2016 & $\begin{array}{l}\text { 18 September } \\
2017\end{array}$ \\
\hline O-RING - 4511913 & $\begin{array}{lr}14 & \text { August } \\
2016 & \\
\end{array}$ & $\begin{array}{l}\text { 18 September } \\
2017\end{array}$ \\
\hline O-RING - 4511912 & $\begin{array}{lr}14 & \text { August } \\
2016 & \end{array}$ & $\begin{array}{l}\text { 18 September } \\
2017\end{array}$ \\
\hline $\begin{array}{l}\text { O-RING } \\
\text { EN17M340 }\end{array}$ & 21 July 2016 & $\begin{array}{lr}25 & \text { August } \\
2017 & \end{array}$ \\
\hline O-RING - 4183312 & 30 June 2016 & $\begin{array}{lr}04 & \text { August } \\
2017 & \end{array}$ \\
\hline $\begin{array}{l}\text { O-RING } \\
\text { EN17M365 }\end{array}$ & 21 July 2016 & $\begin{array}{ll}25 & \text { August } \\
2017 & \end{array}$ \\
\hline PISTON RING & 21 July 2016 & $\begin{array}{lr}25 & \text { August } \\
2017 & \end{array}$ \\
\hline $\begin{array}{l}\text { SEAL RING } \\
4184389\end{array}$ & 21 July 2016 & $\begin{array}{ll}25 & \text { August } \\
2017 & \end{array}$ \\
\hline $\begin{array}{l}\text { SEAL RING - } \\
4184390\end{array}$ & 21 July 2016 & $\begin{array}{lr}25 & \text { August } \\
2017 & \end{array}$ \\
\hline SPACER RING & 21 July 2016 & $\begin{array}{ll}25 & \text { August } \\
2017 & \end{array}$ \\
\hline O-RING - 4181145 & $\begin{array}{ll}07 & \text { January } \\
2016 & \end{array}$ & $\begin{array}{ll}17 & \text { March } \\
2018 & \end{array}$ \\
\hline O-RING - 4181146 & $\begin{array}{l}17 \text { December } \\
2015\end{array}$ & $\begin{array}{l}24 \text { February } \\
2018\end{array}$ \\
\hline O-RING - 4183002 & $\begin{array}{l}17 \text { December } \\
2015\end{array}$ & $\begin{array}{l}24 \text { February } \\
2018\end{array}$ \\
\hline SLIDE VALVE ASS & $\begin{array}{l}07 \text { January } \\
2016\end{array}$ & $\begin{array}{ll}17 & \text { March } \\
2018 & \end{array}$ \\
\hline $\begin{array}{l}\text { SPINDLE GUIDE } \\
\text { ASS }\end{array}$ & $\begin{array}{lr}07 & \text { January } \\
2016 & \end{array}$ & $\begin{array}{ll}17 & \text { March } \\
2018 & \end{array}$ \\
\hline SPACER RING & $\begin{array}{lr}26 & \text { August } \\
2018 & \end{array}$ & $\begin{array}{l}12 \text { January } \\
2023\end{array}$ \\
\hline SCRAPER RING & $\begin{array}{lr}02 & \text { August } \\
2018 & \end{array}$ & $\begin{array}{l}19 \text { December } \\
2022\end{array}$ \\
\hline O-RING - 4181145 & 12 July 2018 & $\begin{array}{l}28 \text { November } \\
2022\end{array}$ \\
\hline
\end{tabular}




\begin{tabular}{|c|c|c|}
\hline Spare part's name & \multicolumn{2}{|c|}{$\begin{array}{c}\text { Date when spare part starts to be } \\
\text { ordered }\end{array}$} \\
\hline O-RING - 4181452 & $\begin{array}{ll}02 & \text { August } \\
2018 & \end{array}$ & $\begin{array}{l}19 \text { December } \\
2022\end{array}$ \\
\hline O-RING - 4181455 & $\begin{array}{ll}02 & \text { August } \\
2018 & \end{array}$ & $\begin{array}{l}19 \text { December } \\
2022\end{array}$ \\
\hline SLIDE VALVE & $\begin{array}{ll}25 & \text { August } \\
2018 & \end{array}$ & $\begin{array}{l}11 \text { January } \\
2023\end{array}$ \\
\hline SPRING & $\begin{array}{ll}25 & \text { August } \\
2018 & \end{array}$ & $\begin{array}{ll}11 & \text { January } \\
2023 & \end{array}$ \\
\hline THRUST PIECE & $\begin{array}{lr}25 & \text { August } \\
2018 & \\
\end{array}$ & $\begin{array}{l}11 \text { January } \\
2023\end{array}$ \\
\hline $\begin{array}{l}\text { CYLINDER COM- } \\
\text { PLETE }\end{array}$ & $\begin{array}{ll}21 & \text { August } \\
2018 & \end{array}$ & $\begin{array}{l}07 \text { January } \\
2023\end{array}$ \\
\hline
\end{tabular}

TABLE III. The date of spare part's ordering for cylinder 2 .

\begin{tabular}{|c|c|c|}
\hline \multirow{2}{*}{$\begin{array}{l}\text { Spare part's name } \\
\text { CRANKPIN BEAR- } \\
\text { ING SHELL }\end{array}$} & \multicolumn{2}{|c|}{$\begin{array}{c}\text { Date when spare part starts to be } \\
\text { ordered }\end{array}$} \\
\hline & $\begin{array}{ll}18 & \text { January } \\
2020 & \end{array}$ & 05 June 2024 \\
\hline $\begin{array}{l}\text { CROSSHEAD } \\
\text { BEARING SHELL }\end{array}$ & $\begin{array}{ll}05 & \text { January } \\
2020 & \end{array}$ & 23 May 2024 \\
\hline O-RING & 25 June 2016 & $\begin{array}{l}\text { 03 September } \\
2018\end{array}$ \\
\hline $\begin{array}{ll}\text { SCRAPER } & \text { RING } \\
\text { (LOWER) } & \end{array}$ & 25 June 2016 & $\begin{array}{l}03 \text { September } \\
2018\end{array}$ \\
\hline $\begin{array}{ll}\text { SCRAPER } & \text { RING } \\
\text { (UPPER) } & \end{array}$ & 25 June 2016 & $\begin{array}{l}\text { 03 September } \\
2018\end{array}$ \\
\hline $\begin{array}{l}\text { TIGHTENING } \\
\text { RING }\end{array}$ & 25 June 2016 & $\begin{array}{l}03 \text { September } \\
2018\end{array}$ \\
\hline $\begin{array}{lll}\text { PISTON } & \text { RING } & - \\
3169804 & & \end{array}$ & 16 July 2016 & $\begin{array}{l}\text { 24 September } \\
2018\end{array}$ \\
\hline $\begin{array}{lll}\text { PISTON } & \text { RING } & - \\
3169805 & & \end{array}$ & 10 May 2016 & 19 July 2018 \\
\hline GUIDE RING & $\begin{array}{l}09 \text { October } \\
2015\end{array}$ & $\begin{array}{l}12 \text { November } \\
2016\end{array}$ \\
\hline O-RING - 4511913 & $\begin{array}{l}17 \text { November } \\
2015\end{array}$ & $\begin{array}{l}21 \text { December } \\
2016\end{array}$ \\
\hline O-RING - 4511912 & $\begin{array}{l}17 \text { November } \\
2015\end{array}$ & $\begin{array}{l}21 \text { December } \\
2016\end{array}$ \\
\hline $\begin{array}{l}\text { O-RING } \\
\text { EN17M340 }\end{array}$ & $\begin{array}{l}24 \text { October } \\
2015\end{array}$ & $\begin{array}{l}27 \text { November } \\
2016\end{array}$ \\
\hline O-RING - 4183312 & $\begin{array}{ll}03 & \text { October } \\
2015 & \end{array}$ & $\begin{array}{l}06 \text { November } \\
2016\end{array}$ \\
\hline $\begin{array}{l}\text { O-RING } \\
\text { EN17M365 }\end{array}$ & $\begin{array}{ll}24 & \text { October } \\
2015 & \end{array}$ & $\begin{array}{l}27 \text { November } \\
2016\end{array}$ \\
\hline PISTON RING & $\begin{array}{ll}24 & \text { October } \\
2015 & \end{array}$ & $\begin{array}{l}27 \text { November } \\
2016\end{array}$ \\
\hline $\begin{array}{lll}\text { SEAL } & \text { RING } \quad- \\
4184389 & & \end{array}$ & $\begin{array}{ll}24 & \text { October } \\
2015 & \end{array}$ & $\begin{array}{l}27 \text { November } \\
2016\end{array}$ \\
\hline $\begin{array}{l}\text { SEAL RING } \quad- \\
4184390\end{array}$ & $\begin{array}{ll}24 & \text { October } \\
2015 & \end{array}$ & $\begin{array}{l}27 \text { November } \\
2016\end{array}$ \\
\hline SPACER RING & $\begin{array}{l}24 \text { October } \\
2015\end{array}$ & $\begin{array}{l}27 \text { November } \\
2016\end{array}$ \\
\hline
\end{tabular}

\begin{tabular}{|c|c|c|}
\hline Spare part's name & \multicolumn{2}{|c|}{$\begin{array}{c}\text { Date when spare part starts to be } \\
\text { ordered }\end{array}$} \\
\hline O-RING - 4181145 & $\begin{array}{ll}07 & \text { January } \\
2016 & \end{array}$ & $\begin{array}{ll}17 & \text { March } \\
2018 & \end{array}$ \\
\hline O-RING - 4181146 & $\begin{array}{l}17 \text { December } \\
2015\end{array}$ & $\begin{array}{l}24 \text { February } \\
2018\end{array}$ \\
\hline O-RING - 4183002 & $\begin{array}{l}17 \text { December } \\
2015\end{array}$ & $\begin{array}{ll}17 & \text { March } \\
2018 & \end{array}$ \\
\hline SLIDE VALVE ASS & $\begin{array}{l}07 \text { January } \\
2016\end{array}$ & $\begin{array}{ll}17 & \text { March } \\
2018 & \end{array}$ \\
\hline $\begin{array}{l}\text { SPINDLE } \\
\text { ASS }\end{array}$ & $\begin{array}{ll}07 & \text { January } \\
2016 & \end{array}$ & $\begin{array}{ll}17 & \text { March } \\
2018 & \end{array}$ \\
\hline SPACER RING & $\begin{array}{l}13 \text { February } \\
2016\end{array}$ & 01 July 2020 \\
\hline SCRAPER RING & $\begin{array}{ll}20 & \text { January } \\
2016 & \end{array}$ & 2020 \\
\hline O-RING - 4181145 & $\begin{array}{l}30 \text { December } \\
2015\end{array}$ & 17 May 2020 \\
\hline O-RING - 4181452 & $\begin{array}{ll}20 & \text { January } \\
2016 & \end{array}$ & 07 June 2020 \\
\hline O-RING - 4181455 & $\begin{array}{ll}20 & \text { January } \\
2016 & \end{array}$ & 07 June 2020 \\
\hline SLIDE VALVE & $\begin{array}{l}12 \text { February } \\
2016\end{array}$ & 30 June 2020 \\
\hline SPRING & $\begin{array}{l}12 \text { February } \\
2016\end{array}$ & 30 June 2020 \\
\hline THRUST PIECE & $\begin{array}{l}12 \text { February } \\
2016\end{array}$ & 30 June 2020 \\
\hline $\begin{array}{l}\text { CYLINDER COM- } \\
\text { PLETE }\end{array}$ & $\begin{array}{l}08 \text { February } \\
2016\end{array}$ & 26 June 2020 \\
\hline
\end{tabular}

TABLE IV. The date of spare part's ordering for cylinder 3.

\begin{tabular}{|c|c|c|}
\hline Spare part's name & \multicolumn{2}{|c|}{$\begin{array}{c}\text { Date when spare part starts to be } \\
\text { ordered }\end{array}$} \\
\hline $\begin{array}{l}\text { CRANKPIN BEAR- } \\
\text { ING SHELL }\end{array}$ & $\begin{array}{lr}23 & \text { August } \\
2018 & \end{array}$ & $\begin{array}{ll}09 & \text { January } \\
2023 & \end{array}$ \\
\hline $\begin{array}{l}\text { CROSSHEAD } \\
\text { BEARING SHELL }\end{array}$ & $\begin{array}{lr}10 & \text { August } \\
2018 & \end{array}$ & $\begin{array}{l}27 \text { December } \\
2022\end{array}$ \\
\hline O-RING & 03 Мау 2016 & 12 July 2018 \\
\hline $\begin{array}{l}\begin{array}{l}\text { SCRAPER } \\
\text { (LOWER) }\end{array} \\
\text { RING }\end{array}$ & 03 May 2016 & 12 July 2018 \\
\hline $\begin{array}{ll}\text { SCRAPER } & \text { RING } \\
\text { (UPPER) } & \end{array}$ & 03 May 2016 & 12 July 2 \\
\hline $\begin{array}{l}\text { TIGHTENING } \\
\text { RING }\end{array}$ & 03 May 2016 & 12 July 20 \\
\hline $\begin{array}{lll}\text { PISTON } & \text { RING } \\
3169804 & \end{array}$ & 24 May 2016 & $\begin{array}{ll}02 & \text { August } \\
2018 & \end{array}$ \\
\hline $\begin{array}{lll}\text { PISTON } & \text { RING } & - \\
3169805 & & \end{array}$ & $\begin{array}{ll}18 & \text { March } \\
2016 & \end{array}$ & 27 May 2018 \\
\hline GUIDE RING & 12 June 2016 & 17 July 2017 \\
\hline O-RING - 4511913 & 21 July 2016 & $\begin{array}{ll}25 & \text { August } \\
2017 & \end{array}$ \\
\hline O-RING - 4511912 & 21 July 2016 & $\begin{array}{ll}25 & \text { August } \\
2017 & \\
\end{array}$ \\
\hline
\end{tabular}




\begin{tabular}{|c|c|c|}
\hline Spare part's name & \multicolumn{2}{|c|}{$\begin{array}{c}\text { Date when spare part starts to be } \\
\text { ordered }\end{array}$} \\
\hline $\begin{array}{l}\text { O-RING } \\
\text { EN17M340 }\end{array}$ & 27 June 2016 & $\begin{array}{ll}01 & \text { August } \\
2017 & \end{array}$ \\
\hline O-RING - 4183312 & 06 June 2016 & 11 July 2017 \\
\hline $\begin{array}{l}\text { O-RING } \\
\text { EN17M365 }\end{array}$ & 27 June 2016 & $\begin{array}{ll}01 & \text { August } \\
2017 & \\
\end{array}$ \\
\hline PISTON RING & 27 June 2016 & $\begin{array}{ll}01 & \text { August } \\
2017 & \end{array}$ \\
\hline $\begin{array}{lll}\text { SEAL } & \text { RING } \quad- \\
4184389 & & \end{array}$ & 27 June 2016 & $\begin{array}{ll}01 & \text { August } \\
2017 & \end{array}$ \\
\hline $\begin{array}{lll}\text { SEAL } & \text { RING } \quad- \\
4184390 & & \end{array}$ & 27 June 2016 & $\begin{array}{ll}01 & \text { August } \\
2017 & \end{array}$ \\
\hline SPACER RING & 27 June 2016 & $\begin{array}{ll}01 & \text { August } \\
2017 & \end{array}$ \\
\hline O-RING - 4181145 & $\begin{array}{lr}16 & \text { January } \\
2016 & \end{array}$ & $\begin{array}{ll}26 & \text { March } \\
2018 & \\
\end{array}$ \\
\hline O-RING - 4181146 & $\begin{array}{l}26 \text { December } \\
2015\end{array}$ & $\begin{array}{ll}05 & \text { March } \\
2018 & \end{array}$ \\
\hline O-RING - 4183002 & $\begin{array}{l}26 \text { December } \\
2015\end{array}$ & $\begin{array}{ll}05 & \text { March } \\
2018 & \end{array}$ \\
\hline SLIDE VALVE ASS & $\begin{array}{ll}16 & \text { January } \\
2016 & \end{array}$ & $\begin{array}{ll}26 & \text { March } \\
2018 & \\
\end{array}$ \\
\hline $\begin{array}{l}\text { SPINDLE GUIDE } \\
\text { ASS }\end{array}$ & $\begin{array}{ll}16 & \text { January } \\
2016 & \end{array}$ & $\begin{array}{ll}26 & \text { March } \\
2018 & \end{array}$ \\
\hline SPACER RING & $\begin{array}{ll}26 & \text { August } \\
2018 & \\
\end{array}$ & $\begin{array}{lr}12 & \text { January } \\
2023 & \end{array}$ \\
\hline SCRAPER RING & $\begin{array}{ll}02 & \text { August } \\
2018 & \end{array}$ & $\begin{array}{l}19 \text { December } \\
2022\end{array}$ \\
\hline O-RING - 4181145 & 12 July 2018 & $\begin{array}{l}28 \text { November } \\
2022\end{array}$ \\
\hline O-RING - 4181452 & $\begin{array}{ll}02 & \text { August } \\
2018 & \\
\end{array}$ & $\begin{array}{l}19 \text { December } \\
2022\end{array}$ \\
\hline O-RING - 4181455 & $\begin{array}{ll}02 & \text { August } \\
2018 & \end{array}$ & $\begin{array}{l}19 \text { December } \\
2022\end{array}$ \\
\hline SLIDE VALVE & $\begin{array}{ll}25 & \text { August } \\
2018 & \\
\end{array}$ & $\begin{array}{ll}11 & \text { January } \\
2023 & \\
\end{array}$ \\
\hline SPRING & $\begin{array}{ll}25 & \text { August } \\
2018 & \\
\end{array}$ & $\begin{array}{ll}11 & \text { January } \\
2023 & \end{array}$ \\
\hline THRUST PIECE & $\begin{array}{ll}25 & \text { August } \\
2018 & \\
\end{array}$ & $\begin{array}{ll}11 & \text { January } \\
2023 & \\
\end{array}$ \\
\hline $\begin{array}{l}\text { CYLINDER COM- } \\
\text { PLETE }\end{array}$ & $\begin{array}{ll}21 & \text { August } \\
2018 & \\
\end{array}$ & $\begin{array}{ll}07 & \text { January } \\
2023 & \\
\end{array}$ \\
\hline
\end{tabular}

TABLE V. The date of spare part's ordering for cylinder 4.

\section{CONCLUSIONS}

Petri net can be used to represent a process of ship engine component's spare part ordering. Based on the Petri net model, we can build a max-plus-algebra model to find the maximal time of spare part ordering. Therefore, we obtain the date when the spare part should be ordered.

\section{REFERENCES}

[1] Subiono, Aljabar Min-Max-Plus dan Terapannya. Surabaya: Institut Teknologi Sepuluh Nopember, 2015.
[2] D. Indriyani and Subiono, "Scheduling of the crystal sugar production system in sugar factory using max-plus algebra," International Journal of Computing Science and Applied Mathematics, vol. 2, no. 3, pp. 33-37, 2016.

[3] F. Baccelli, G. Cohen, G. Olsder, and J.-P. Quadrat, Synchronization and linearity: an algebra for discrete event systems. John Wiley \& Sons Ltd, 1992.

[4] Subiono and K. Fahim, "On computing supply chain scheduling using max-plus algebra," Applied Mathematical Sciences, vol. 10, no. 10, pp. 477-486, 2016. 